\title{
Geometric properties and charged particles yields behind Glauber model in high energy $p A$ and AA collisions
}

\author{
Tatiana Drozhzhova \\ Saint Petersburg State University, Russia \\ E-mail: tatiana.drozhzhova@cern.ch \\ Grigory Feofilov \\ Saint Petersburg State University, Russia \\ E-mail: feofilov@hiex.phys.spbu.ru
}

\section{Vladimir Kovalenko}

Saint Petersburg State University, Russia

E-mail: nvkinf@rambler.ru

\section{Andrey Seryakov ${ }^{1}$}

Saint Petersburg State University, Russia

E-mail: seryakov@yahoo.com

\begin{abstract}
We present two independent estimates of the number of nucleon-nucleon collisions $\left(N_{\text {coll }}\right)$ and the number of participant nucleons $\left(N_{\text {part }}\right)$ in AA and pA interactions at the LHC energies, obtained using Monte Carlo calculations: the Modified Glauber model (MGM) and more detailed "non-Glauber Model", based on the color dipoles approach. Contrary to generally used standard Glauber model, the MGM takes into account in an effective way the energymomentum conservation in soft particles production. Thus it provides with only one parameter the quantitative description of scaling of the charged particle yields with $N_{\text {part }}$, describing observed in experiment in a wide energy region from SPS to LHC. The second model provides parton-string description of pp, pA and AA interaction considering the energy conservation in nucleon-nucleon collisions and string interaction (string fusion). These estimates are confirmed by calculations using the MC event generators (AMPT and HIJING). The results are pointing at the effect of noticeable stopping of nucleons in $\mathrm{pA}$ and AA collisions. Taking into account the obtained considerable decrease of $N_{\text {coll }}$ and $N_{\text {part }}$ in pA collisions, compared to Glauber values, we suggest reconsidering the use of Glauber normalization in determination of charged-particles pseudorapidity densities in $\mathrm{p}-\mathrm{Pb}$ collisions at LHC energies.
\end{abstract}

The XXI International Workshop High Energy Physics and Quantum Field Theory June 23 - June 30, 2013

Saint Petersburg Area, Russia

${ }^{1}$ Speaker 


\section{Introduction}

The energy density, achieved in the region of interaction in hadron-hadron collisions, is one of the most important parameters characterizing the collision process. Sufficiently high values of this veriable, exceeding some critical value, are needed to study the formation and properties of new physical states of matter, such as quark-gluon plasma. The intuitive picture of the energy density in hadron-hadron collisions is based on the common use of geometrical factors such as the impact parameter and nuclear matter density profile of the given colliding systems. The region of interaction is usually defined in this approach by some observables related, in case of nucleus-nucleus collisions, to the energy (and number) of participating nucleons $\left(N_{\text {part }}\right)$. The last ones could be measured using calorimeters, installed on the collision axis (so-called Zero-degree calorimeters) that measure the energy of forward flying spectatornucleons $\left(N_{\text {spec }}\right)$. Then in case of collisions between A and B nuclei the number of nucleonparticipants is given by $N_{\text {part }}=A+B-N_{\text {spec }}$, where $A$ and $B$ are relevant mass numbers, and $N_{\text {spec }}$ is the total number of spectators from both nuclei. However, the measurements of $N_{\text {spec }}$ are quite demanding and in the overwhelming majority of present experiments the concept of the standard Glauber model is being used for the estimations of $N_{\text {part }}$. In such approach the classes of events are selected using the predefined, fixed values in percentiles from the distributions of some measured observables like charged particles multiplicity (see e.g. [1, 2]). Fitting of the multiplicity distributions is performed within standard Glauber approach using some arbitrary multiplicity scaling factor. Thus, the relation between the multiplicity class and the impact parameter and values of $N_{\text {part }}$ is introduced. In this approach the energy density reached for the given centrality class in the interaction region is relevant to number of nucleonnucleon collisions $\left(N_{\text {coll }}\right)$. The last one is a pure model quantity and could be strongly biased by the theoretical assumptions. In particular, the purely geometrical Glauber picture of nucleusnucleus or proton-nucleus collision does not take into account any energy losses in any of multiple nucleon-nucleon collisions, leading to the overestimates of $N_{\text {coll }}$. The present report is devoted to question of legitimacy of the standard Glauber model for the estimates of centrality and such variables as $N_{\text {coll }}$. in $\mathrm{Pb}-\mathrm{Pb}$ and p- $\mathrm{Pb}$ collisions at the LHC energies. In the first section we continue the analysis in the framework of the Modified Glauber model with the account of the energy-momentum conservation in soft particles production (MGM) [3]. In the second section the parton-string description of pp, $\mathrm{pA}$ and $\mathrm{AA}$ interaction is presented considering the energy conservation in nucleon-nucleon collisions and string interaction in a form of string fusion. Finally we compare our results to those of some MC event-generators and discuss the use of standard Glauber normalization in determination of charged-particles pseudorapidity densities in $\mathrm{p}-\mathrm{Pb}$ collisions at LHC.

\section{Modified Glauber Model: p-Pb collisions at the LHC}

Standard Glauber model is extensively used to compute the number of participants, the number of collisions and their variances [3], as a base for the analysis of data coming from multiple hadron production in high energy nucleus-nucleus collisions from AGS to SPS and 
RHIC energies. Previously (see [3]), we modified the standard Glauber model calculations of number of participants $\left(N_{\text {part }}\right)$, number of collisions $\left(N_{\text {coll }}\right)$ and their variances using a simplified estimate of the energy loss in any nucleon-nucleon collision. A fraction equal to $1-k$ of the initial nucleon momentum was allowed to be lost in each inelastic interaction, so that two "nucleon-like structures", emerging after the nucleon-nucleon inelastic process, have lower momenta. Therefore, further "nucleon-nucleon" inelastic collisions take place at a lower energy $\sqrt{s}$ and with the experimentally known cross-section $\left(\sigma_{\text {inel }}^{\mathrm{pp}}(\sqrt{s})\right)$. For each particular step in nucleon-nucleon collisions the charged particles mean multiplicity value was taken from pp data at the given value $\sqrt{s}$. As a results, a considerable reduction in the number of collisions $\left(N_{\text {coll }}\right)$ and in the total mean number of charged particles $\left(N_{\mathrm{ch}}\right)$, compared to the standard Glauber approach was obtained, while the number of participants $\left(N_{\text {part }}\right)$ was practically unchanged. The new developments of the MGM [4] included the account of the nucleon repulsive core and more detailed analysis of interaction of secondaries, these effects were found of some importance for the peripheral collisions (see Fig. 3).

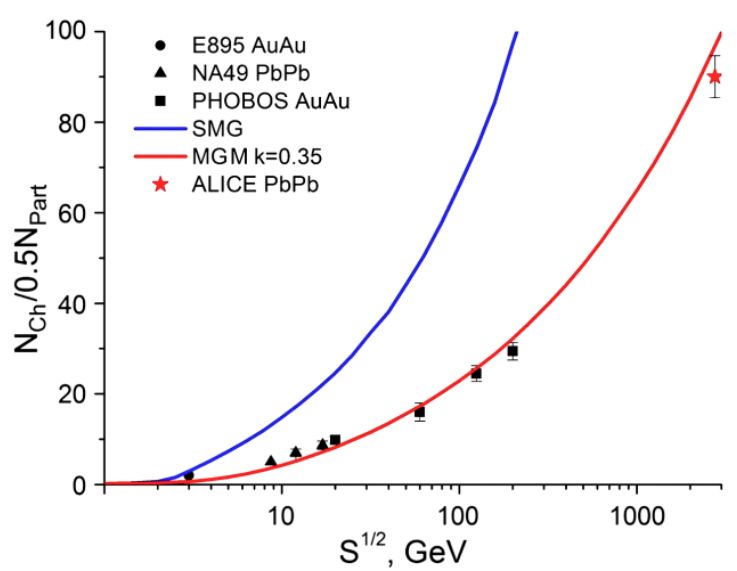

Figure 1: Average total multiplicity of charged particles for the most central AA collisions, related to a pair of nucleon-participants as a function of $\sqrt{s}$ : blue line - the Glauber model (SGM), red line - the Modified Glauber model (MGM, $k=0.35$ ), fitted up to $200 \mathrm{GeV}$ and calculated up to to $\sqrt{s}=2.76 \mathrm{TeV}$. Black points experimental data $[5,6]$. Red star - ALICE data [7].

We used this last version [4] of the Modified Glauber Model (MGM) in the present calculations. A single parameter $k$ was defined by fitting the available data on the average total charged particles multiplicity yields in AA collisions vs $\sqrt{s}$. Results are shown in the Fig.1. The same MGM parameter $k=0.35$ was used in the data description in Fig. 2, where the comparison of the MGM to the experimental data [5, 6, 7] on the normalized charged particle yields is shown at different centralities (defined by $N_{\text {part }}$ values). One may see that this model with the simplified assumption of energy-momentum conservation in multiparticle production provides a reasonably good description of experimental data in a wide energy range from the ISR to the LHC. We use the same value of the MGM parameter $k=0.35$ in our analysis of data for $\mathrm{p}-\mathrm{Pb}$ collisions at the LHC energy. The results of our estimates of $\left\langle N_{\text {part }}\right\rangle$ in minimum bias $\mathrm{p}-\mathrm{Pb}$ collisions in MGM approach are compared in the Table 1 to Glauber, to string-fusion nonGlauber partonic level approaches and to MC event generators calculations. 


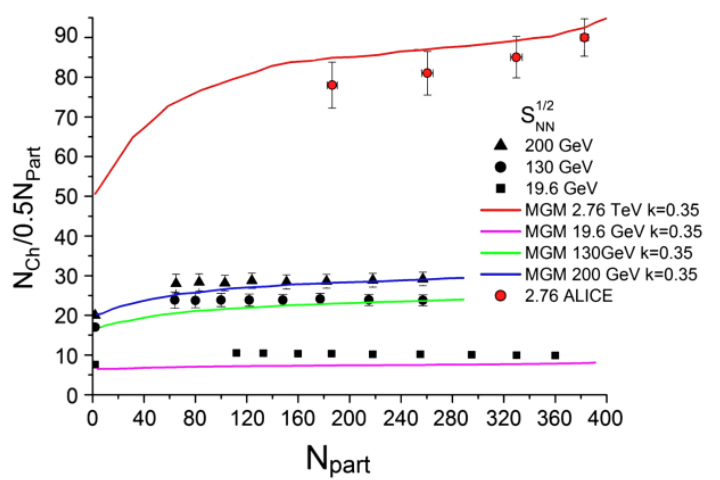

Figure 2: MGM predictions $(\mathrm{k}=0.35)$ of the total charged-particle multiplicity vs $N_{\text {part }}$ in high-energy heavy ion collisions in comparison with $\mathrm{Pb}-\mathrm{Pb}, \mathrm{Au}-\mathrm{Au}$ and $\mathrm{pp}(\mathrm{p} \overline{\mathrm{p}})$ data $[5,6,8]$.

\section{The parton-string description of pp, pA and $A A$ interactions}

The Non-Glauber approach $[9,10]$ is based on the partonic description of nucleons interaction. Initially the nucleons, filling the nucleus according to Woods-Saxon distribution, are thought to consist of certain number quark-diquark and quark-antiquark pairs. The latter are distributed in the transverse plane around the center of each nucleon according to Gaussian function. For each parton the appropriate momentum fraction is assigned, according to the exclusive distributions [9], accounting the energy and angular momentum conservation in the initial state of a nucleon.

Each quark-diquark and quark-antiquark pair is supposed to form of a dipole. The probability of elementary collision of two dipoles with transverse coordinates $\left(\vec{r}_{1}, \vec{r}_{2}\right)$ and $\left(\vec{r}_{1}^{\prime}, \vec{r}_{2}{ }^{\prime}\right)$ is given by the amplitude with confinement effects taken into account:

$$
f=\frac{\alpha_{s}}{2}\left[K_{0}\left(\frac{\left|\vec{r}_{1}-\vec{r}_{1}^{\prime}\right|}{r_{\max }}\right)+K_{0}\left(\frac{\left|\vec{r}_{2}-\vec{r}_{2}^{\prime}\right|}{r_{\max }}\right)-K_{0}\left(\frac{\left|\vec{r}_{1}-\vec{r}_{2}^{\prime}\right|}{r_{\max }}\right)-K_{0}\left(\frac{\left|\vec{r}_{2}-\vec{r}_{1}^{\prime}\right|}{r_{\max }}\right)\right]^{2},
$$

where $K_{0}$ is a modified Bessel function, $\alpha_{s}$ is an effective coupling constant, and

$r_{\text {max }} \simeq 0.2-0.3 \mathrm{fm}$ is characteristic confinement scale, the exact values are turned by fitting to the experimental data on multiplicity.

Important, that in this approach every parton can interact with other one only once, forming a pair of quark-gluon strings, producing particles, thus the energy conservation in nucleons collision is fulfilled explicitly [13]. The charged particles multiplicity is calculated in the approach of color strings, taking into account their finite rapidity width and the hypothesis of interactions between them (in a form of string fusion). The latter could be considered due to non-zero transverse radius of string $r_{\text {str }}$ (string fusion) [11], and is implemented by introducing a lattice in the transverse plane [12].

Parameters of the model are constrained from the pp data on the total inelastic cross section and on charged multiplicity in wide energy range (from ISR to LHC) with the additional requirement of consistent multiplicity in minimum bias p-Pb collisions at the LHC [14].

In the Fig. 3 the results of the calculations in MC model of pseudorapidity multiplicity density in $\mathrm{Pb}-\mathrm{Pb}$ collisions at $2.76 \mathrm{TeV}$ with varius parameter combinations are compared with the experimental data. The set of parameters with $r_{\mathrm{str}}=0.2-0.3 \mathrm{fm}$ consistently describing the multiplicity in $\mathrm{pp}, \mathrm{p}-\mathrm{Pb}$ and $\mathrm{Pb}-\mathrm{Pb}$ collisions, was used to determine the uncertainties of the model. One may see the case without string fusion is completely excluded by the experimental data. 

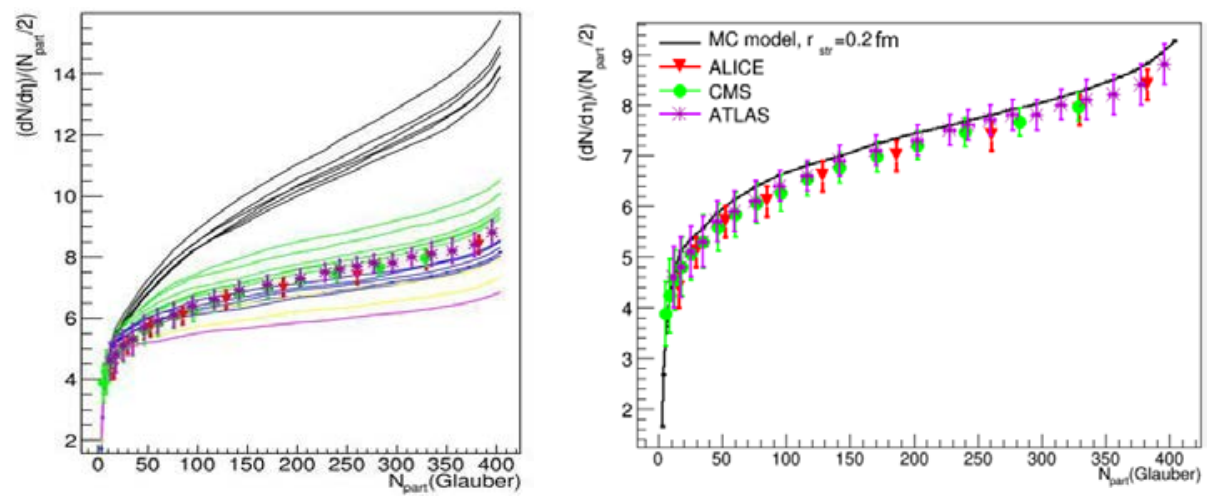

Figure 3: Charged particle dendities in Pb-Pb collisions at the LHC vs. centrality. Left: the data points are measured by ALICE (red traingles) [1], ATLAS (violet stars) [15] and CMS (green circles) [16]; lines - various sets of parameters of the non-Glauber MC string fusion model [14]. The upper set of curves is without string fusion. The lower set includes string fusion. Right: the non-Glauber MC string fusion model with string radius $r_{\text {str }}=0.2 \mathrm{fm}$ in comparison with data.

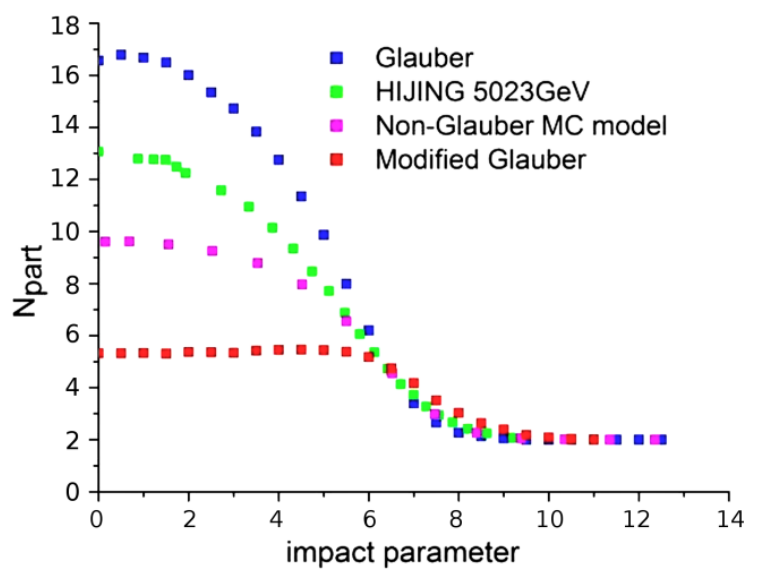

Figure 4: $N_{\text {part }}$ vs. impact parameter (in fm) for p-Pb collisions at the LHC: Glauber, MGM, Non-Glauber [13] obtained in the present work in comparison with our HIJING 2.0 calculation with gluon shadowing parameter $\mathrm{s}_{\mathrm{g}}=0.20-0.23$.

\section{3.pA and AA interactions in MC event generators}

HIJING2.0 [18] calculations with gluon shadowing (parameter $\mathrm{s}_{\mathrm{g}}=0.20-0.23$ ) were compared succesfully in [21] to combined data on charged particle yields vs centrality in Au-Au collisions at RHIC [20] and to ALICE data on rapidity distributions in p-Pb collisions at the LHC [17]. Results of Fig. 4 for $N_{\text {part }}$ vs. impact parameter, which we obtained using HIJING calculations, show that the energy-momentum conservation in HIJING with gluon shadowing gives as a result remarkably smaller number of $N_{\text {part }}$ compared to standard Glauber calculations. The relevant mean number of $\left\langle N_{\text {part }}\right\rangle$, calculated in HIJING for the minimum bias data is shown in the Tbale 1. Quite similar to HIJING results on $\left\langle N_{\text {part }}\right\rangle$ in p-Pb are obtained in AMPT event-generator [19] (see Table 1.). Thus we may conclude here that our calculations of $\left\langle N_{\text {part }}\right\rangle$ in minimum bias p-Pb collisions at the LHC in MGM and Non-Glauber approaches (see 
Table 1) are supported by the data form the MC event-generators, based on different approaches. Therefore, taking into account the obtained considerable decrease of $N_{\text {part }}$ in pA collisions, compared to Glauber values, we suggest to reconsider the use of Glauber normalization in determination of charged-particles pseudorapidity densities in p-Pb collisions. This statement is also relevant for a broad energy range, including the energies below the LHC. Fig. 4 shows the compilation of data on $\mathrm{pp}, \mathrm{p}-\mathrm{Pb}, \mathrm{Au}-\mathrm{Au}$ and $\mathrm{Pb}-\mathrm{Pb}$ collisions at different energies including the latest LHC data [17] on $\mathrm{p}-\mathrm{Pb}$ charged particle yields normalized to Glauber-defined $\left\langle N_{\text {part }}\right\rangle$. Results of application of different approaches, using the MGM and Non-Glauber models, are indicated at the LHC energy by two arrows.

\begin{tabular}{|l|l|}
\hline Models & Value of $\left\langle\mathrm{N}_{\text {part }}\right\rangle$ in p-Pb at $5.02 \mathrm{TeV}$ \\
\hline Glauber & $7.9 \pm 0.6(\mathrm{ALICE}[17])$ \\
\hline MGM & $4.3 \pm 0.3$ \\
\hline Non-Glauber [13] & $6.2 \pm 0.6$ \\
\hline HIJING & 6.5 \\
\hline AMPT [4] & 6.4 \\
\hline
\end{tabular}

Table 1: Estimates of $\left\langle N_{\text {part }}\right\rangle$ in minimum bias p-Pb collisions in Glauber and non-Glauber approaches of (note that $\left\langle N_{\text {coll }}\right\rangle=\left\langle N_{\text {part }}\right\rangle-1$ ).

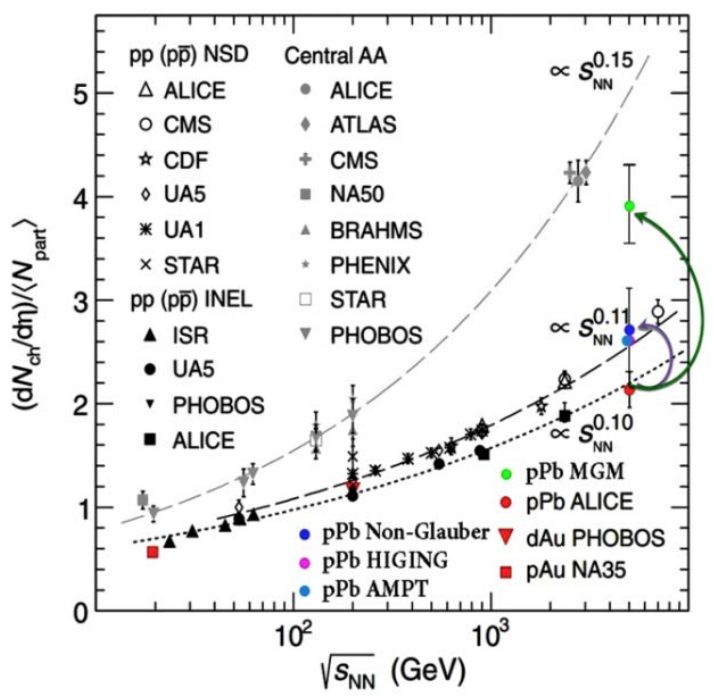

Figure 5: Charged-particle pseudorapidity density at midrapidity normalized to the number of participants, calculated with the Glauber model, for $\mathrm{p}-\mathrm{Pb}, \mathrm{p}-\mathrm{Au}$, and d-Au collisions as a function of $\sqrt{S_{N N}}$, superimposed on the NSD pp (pp) and central heavy-ion data, respectively; while NN is superimposed on the inelastic pp ( $p \bar{p})$ data (see refs in [17]). Our corrections, based on different estimates of $\left\langle N_{\text {part }}\right\rangle$ (see Table 1), are shown for p-Pb collisions for the LHC.

\section{Conclusions.}

Results of our calculations show that the proper account of energy-momentum conservation in multiparticle production at the LHC leads to the considerable stopping of nucleons interacting inelastically with each other in $\mathrm{p}-\mathrm{Pb}$ or $\mathrm{Pb}-\mathrm{Pb}$ collision process. Results of our approach, based on two different model estimates (MGM and Non-Glauber), are confirmed 
with MC HIJING and AMPT event generators - all these models gives smaller values of $\left\langle N_{\text {part }}\right\rangle$ in $\mathrm{p}-\mathrm{Pb}$ compared to Glauber. Therefore, we suggest reconsidering the use of Glauber normalization in determination of charged-particles pseudorapidity densities in $\mathrm{p}-\mathrm{Pb}$ collisions at LHC energies.

\section{References}

[1] K. Aamodt et al. (ALICE Collaboration), Phys. Rev. Lett. 106 (2011) 032301.

[2] B. Abelev et al (ALICE Collaboration), Phys. Rev. C 88 (2013) 044909.

[3] G. Feofilov, A. Ivanov, Number of nucleon-nucleon collisions vs. energy in modified Glauber calculations, J. Phys. Conf. Ser. 5 (2005) 230.

[4] A. Seryakov, pA collisions at LHC in Modified Glauber model, in Proceedings of International Student Conference "Science and Progress" (St. Petersburg, Peterhof, 2012), 131.

[5] B. Back et al. (PHOBOS Collaboration), Phys. Rev. Lett. 93 (2004) 082301.

[6] B. Back et al. (PHOBOS Collaboration), Comparison of the Total Charged-Particle Multiplicity in High-Energy Heavy Ion Collisions with $e+e-$ and pp/p(anti)p Data, nucl-ex/0301017v1 (2003).

[7] K. Aamodt et al. (ALICE Collaboration), Phys. Rev. Lett. 105 (2010) 252301.

[8] K. Aamodt et al. (ALICE Collaboration), Centrality dependence of the pseudorapidity density distribution for charged particles in $\mathrm{Pb}$-Pb collisions at $\sqrt{S_{N N}}=2.76 \mathrm{TeV}$, Phys. Lett. B 76 (2013) 610, arXiv:1304.0347 [nucl-ex].

[9] V. N. Kovalenko, Modeling of exclusive parton distributions and long-range rapidity correlations in proton-proton collisions at the LHC energies, Phys. Atom. Nucl. 76 (2013) 1189, arXiv:1211.6209 [hep-ph].

[10] V. Kovalenko, V. Vechernin, Model of pp and AA collisions for the description of long-range correlations, PoS (Baldin ISHEPP XXI) 077 (2012), arXiv:1212.2590 [nucl-th].

[11] M. A. Braun, R. Kolevatov, C. Pajares, V. Vechernin, Eur. Phys. J. C 32 (2004) 535

[12] V. V. Vechernin, R. S. Kolevatov, Phys. Atom. Nucl. 70 (2007) 1797

[13] V. N. Kovalenko. Proton-nucleus collisions at LHC energy in the Monte Carlo model, arXiv:1308.1932 [hep-ph] (2013).

[14] V. Kovalenko, Monte Carlo model for pp, pA and AA collisions at high energy: parameters tuning and results, PoS (QFTHEP 2013) 052 (2013).

[15] G. Aad et al (ATLAS Collaboration), Phys. Lett. B 710 (2012) 363

[16] S. Chatrchyan et al (CMS Collaboration), JHEP 08 (2011) 141

[17] B. Abelev et al. (ALICE Collaboration), Phys. Rev. Lett. 110 (2013) 032301.

[18] R. Xu, W.-T. Deng, and X.-N. Wang, Phys. Rev. C 86 (2012) 051901, arXiv:1204.1998.

[19] J. Albaete, N. Armesto, R. Baier, et al., Int. J. Mod. Phys. E 22 (2013) 1330007

[20] S. S. Adler et al. (PHENIX Collaboration), Phys. Rev. C 71 (2005) 034908 [Erratum-ibid. C 71 (2005) 049901].

[21] Wei-tian Deng, Xin-Nian Wang, Rong Xu, Phys. Lett. B 701 (2011) 133. 